\title{
Relationship between Obesity and Coronary Artery Disease Defined by Coronary Computed Tomography Angiography
}

Lara Luiza Silvello Pereira, ${ }^{\circledR}$ Gisele Marochi de Moraes, ${ }^{1 \oplus}$ Adriano Camargo de Castro Carneiro, ${ }^{2}{ }^{\circledR}$ Valéria de Melo Moreira, ${ }^{\circledR}$ Juliana Hiromi Silva Matsumoto Bello, ${ }^{2 \oplus}$ Carlos Eduardo Elias dos Prazeres, ${ }^{2 \oplus}$ Carlos Eduardo Rochitte, $^{2 ®}$ Tiago Magalhaes ${ }^{2}{ }^{\circledR}$

Pontifícia Universidade Católica do Paraná (PUCPR), ${ }^{2}$ Paraná, PR - Brazil

$H C O R,{ }^{2}$ São Paulo, SP - Brazil

\section{Abstract}

Background: Although associated with traditional cardiovascular risk factors, it is unclear whether obesity alone is associated with coronary artery disease (CAD).

Objective: To investigate the role of obesity as a risk factor for CAD, defined by coronary computed tomography angiography (CCTA).

Methods: This study retrospectively included 1,814 patients referred for CCTA in a hospital in São Paulo, from August 2010 to July 2012. CAD was identified by coronary calcium score and presence of coronary stenosis $>50 \%$. Images were analyzed by two specialists, and the coronary findings were compared between obese and non-obese groups. A multivariate analysis model was used to assess obesity as an independent variable for the occurrence of obstructive CAD.

Results: Among the study population, mean age was $58.5+/-11.5$ years, $22.8 \%$ were obese $\left(B M I=30 \mathrm{~kg} / \mathrm{m}^{2}\right)$ and $66.3 \%$ were male. The prevalence of obstructive CAD was $18.4 \%$ in both groups. Obese patients had higher median calcium score compared to non-obese subjects (14.7 vs. 1.4 , respectively, $\mathrm{p}=0.019)$. In the multivariate analysis, obesity was not an independent factor for obstructive CAD (coefficient $=-0.035, p=0.102$ ).

Conclusion: Although no differences were observed in the prevalence of obstructive CAD between obese and non-obese individuals, coronary calcium scores were significantly in lower the latter group. (Int J Cardiovasc Sci. 2020;33(1):57-64)

Keywords: Coronary Artery Disease; Obesity; Body Mass Index; Dyslipidemias; Risk Factors; Prevalence; Tomography/métodos; Coronary Angiography/methods.

\section{Introduction}

Cardiovascular diseases are the main causes of morbidity and mortality in the world population. Due to aging of the global population, cardiovascular diseases, mainly represented by coronary artery disease (CAD), play an incremental role on global mortality. ${ }^{1}$

In this context, changes in lifestyle have contributed to increased incidence of cardiovascular risk factors and ultimately of coronary disease. Due to its increasing incidence on a global scale (39\% of adults aged 18 years and older are obese), obesity has become one of the factors with the greatest impact on the risk of CAD. ${ }^{2}$

Obesity is recognized as one of the most important underlying risk factors for a wide variety of metabolic diseases, such as hypertension, dyslipidemia, and diabetes, which are strongly associated with the development of cardiovascular diseases. ${ }^{3}$ Nevertheless, whether obesity alone is a risk factor for CAD has not been well established. ${ }^{4-6}$ In this regard, the phenotype 
of metabolically healthy but obese (MHO) individuals, with hormonal and insulin resistance profile not compatible with increased adiposity has become a matter of discussion. ${ }^{7,8}$

Previous studies have investigated the incidence of cardiovascular disease in $\mathrm{MHO}$, with controversial results. ${ }^{9,10}$ Also, although data derived from intermediate markers of disease (e.g. the carotid intima media thickness) can evaluate the association of these parameters with the presence of CAD in MHO individuals, there are few data available about the association between body mass index (BMI) and coronary artery calcium score as determinant of subclinical atherosclerosis. Coronary artery calcium score was shown to be superior than other methods for the evaluation of subclinical atherosclerosis in cardiovascular event prediction. ${ }^{11}$

Therefore, the aim of the present study was to evaluate whether obesity alone is correlated with the presence of $\mathrm{CAD}$, evaluated by coronary computed tomography angiography (CCTA).

Methods

\section{Patients and study design}

We reviewed the database and patients medical records in a tertiary hospital in Sao Paulo (Brazil). The sample was composed of 1,814 patients consecutively referred for cardiac/coronary computed tomography angiography between August 2010 and July 2012.

The study was approved by the ethics committee of the Pontifical Catholic University of Paraná (approval number 1524216) and was in accordance with the Helsinki Declaration. The study was registered in Plataforma Brasil (registration number 55363016.6.0000.0020) and informed consent to participate in this study was waived. All data were collected and registered in specific spreadsheets by trained investigators, and then manually transferred to a database of the CCTA division.

\section{Epidemiological and clinical data}

Data contained in the patient admission questionnaire were collected by direct interview and/or from medical records. Variables included demographic and anthropometric data, indication for CCTA, risk factors for CAD - hypertension, diabetes, dyslipidemia, smoking, family history of CAD - parameters of CCTA acquisition and results of the test.
Computed tomography angiography, a contrast computed tomography, is clinically used for evaluation of coronary stenosis/obstruction. The test allows the calculation of the coronary artery calcium, which consists in a non-invasive imaging method to identify atherosclerosis in asymptomatic individuals.

Definitions of obese and metabolically healthy but obese patients

Patients with a BMI greater than $30 \mathrm{~kg} / \mathrm{m}^{2}$ were considered obese, and MHO patients were identified based on the absence of the following criteria 1) hypertriglyceridemia (triglycerides $>150 \mathrm{mg} / \mathrm{dL}$ ) or pharmacological treatment for this condition; 2) low HDL-cholesterol (HDL $<40 \mathrm{mg} / \mathrm{dL}$ ) or pharmacological treatment for this condition; 3) hypertension, defined as blood pressure $\geq 130 / 85 \mathrm{mmHg}$ or pharmacological treatment for this condition; 4) altered fasting glucose (glucose $\geq 100 \mathrm{mg} / \mathrm{dL}$ ) or diagnosis of diabetes, or pharmacological treatment for this condition.

\section{Coronary computed tomography angiography}

\section{Acquisition parameters and protocol}

Two computed tomography scanners were used for the tests - Siemens Somatom Sensation 64 and Siemens Somaton Definition Flash (Siemens Healthcare, Forchheim, Germany), following respective protocols. Patients with blood pressure higher than $100 \mathrm{mmHg}$ received $5 \mathrm{mg}$ sublingual nitrates prior to the test, whereas a beta-blocker (metoprolol $150 \mathrm{mg}$ in patients with $\mathrm{BMI} \geq 30$ $\mathrm{kg} / \mathrm{m}^{2}$, and $75 \mathrm{mg}$ in those with $\mathrm{BMI} \leq 30 \mathrm{~kg} / \mathrm{m}^{2}$ ) was orally administered to patients with a heart rate higher than $80 \mathrm{bpm}$ on the test day. In addition, if necessary, intravenous metoprolol (maximum $20 \mathrm{mg}$ ) was used during CCTA to achieve target heart rate $(\leq 65 \mathrm{bpm})$.

Patients with no history of angioplasty or surgical revascularization underwent computed tomography scanning synchronized with electrocardiography before contrast injection for quantification of coronary artery calcium (Agatston units). Subsequently, contrast was injected at high flow rates (maximum of $6 \mathrm{~mL} / \mathrm{s}$ Henetix $350 \mathrm{mg} / \mathrm{mL}$, Guerbet, Rio de Janeiro, Brazil), with concomitant acquisition of CCTA. The following parameters were obtained for analysis: 1) tube voltage of $100-140 \mathrm{kV}$; 2 ) adjusted tube current (estimated by the tomography device according to chest attenuation of each patient); 3) collimation $2 \times 128 \times 0.6 \mathrm{~mm}$ or $64 \times 0.6 \mathrm{~mm}$, according to the scanner specifications. The tests on both 
scanners were performed in helical acquisition mode, or in prospective axial and high-pitch spiral mode by the dual-source (two x-ray sources) scanner.

\section{Image reconstruction}

For coronary artery calcium score calculation, images were reconstructed with a section thickness of $3 \mathrm{~mm}$ and $3 \mathrm{~mm}$ - interval. Coronary calcifications with attenuation $\geq 130 \mathrm{HU}$ in an area $\geq 3 \mathrm{~mm}^{2}$ were quantified, according to the algorithm proposed by Agatston et al. ${ }^{12}$

CCTA images were reconstructed with a section thickness of $0.6 \mathrm{~mm}$ and increment of $0.3 \mathrm{~mm}$ in systole and/or automatically or manually determined (in case of spiral or prospective acquisition), to minimize cardiac motion artifacts. For better image quality, iterative reconstruction algorithms were performed.

\section{Image interpretation}

All images (calcium score and CCTA) were analyzed on a dedicated workstation (Leonardo Definition, Siemens Healthcare, Erlanger, Germany). All CCTA images were analyzed by two observers; discrepancies were resolved by consensus.

Coronary artery calcium was quantitively determined by visual identification of coronary calcifications. Lesions in different coronary territories were automatically summed to determine the total calcium score.

Per-segment analysis of CCTA images was performed following the Society of Cardiovascular Computed Tomography guidelines. ${ }^{13}$

CAD was established at two levels: 1 ) calcium score $>0$ (Agatston); 2) presence of atherosclerotic plaque (CCTA). Obstructive coronary disease was defined by the presence of any coronary stenosis $\geq 50 \%$.

\section{Statistical analysis}

Binary data were described in absolute numbers and percentages. Continuous variables with normal distribution were presented as mean and standard deviation, whereas those without a normal distribution were presented as median and interquartile range. Data normality was tested by the Shapiro-Wilk test; coronary artery calcium score was the only variable that was not normally distributed. Categorical variables were compared using the chi-square test. Continuous variables were compared using the unpaired Student's t-test.
Calcium score between obese and non-obese patients was compared by the Mann-Whitney test.

A multiple linear regression model was used to assess the relationship between cardiovascular risk factors and the presence of obstructive CAD. For continuous variables of the model, $\beta$ coefficients were used to indicate changes in the dependent variable (presence of obstructive $\mathrm{CAD}$ ) for a unit change in each independent variable after controlling for confounding variables. For categorical variables (e.g. sex, smoking), $\beta$ coefficient represents the difference in the dependent variable (presence of obstructive CAD) according to the status (e.g. male vs. female; smokers vs. non-smokers) after controlling of confounding variables of the model.

Statistical analysis was performed using the STATA software (version 11, STATACorp, College Station, Texas, USA). The level of significance was set as $5 \%$.

\section{Results}

A total of 1,814 consecutive patients with a medical indication for cardiac/coronary computed tomography angiography, were referred to a tertiary hospital in São Paulo between August 2010 and July 2012. We excluded from analysis patients whose indication for the test was not screening for CAD (e.g. patients with congenital heart disease, patients referred for evaluation of valve disease or pulmonary veins). In addition, we also excluded patients with history of CAD (myocardial infarction, angioplasty and /or surgical myocardial revascularization). A total of 1,383 patients were evaluated (Figure 1). Table 1 describes main epidemiological characteristics of the patients. Mean age was $58.5+/-11.5$ years, and $66.3 \%(n=917)$ of patients were men.

In general, the prevalence of cardiovascular risk factors was not different between obese and non-obese subjects (Table 1), and the same was observed for the prevalence of obstructive CAD. Obstructive CAD was present in a similar percentage (18.4\% in both groups) in obese patients $(\mathrm{n}=58)$ and in those with BMI < $30 \mathrm{~kg} / \mathrm{m}^{2}(\mathrm{n}=197)$ (Figure 2). The presence of CAD, defined by the presence of coronary calcifications, was significantly different between the groups. Median calcium score was 1.4 and 14.7 Agatston units in the groups of non-obese and obese patients, respectively (Figure 2). In our sample, mean calcium score percentile, by age, sex and ethnicity was 61 .

In order to establish the role of each risk factor on the development of obstructive $\mathrm{CAD}$, we used a multiple 


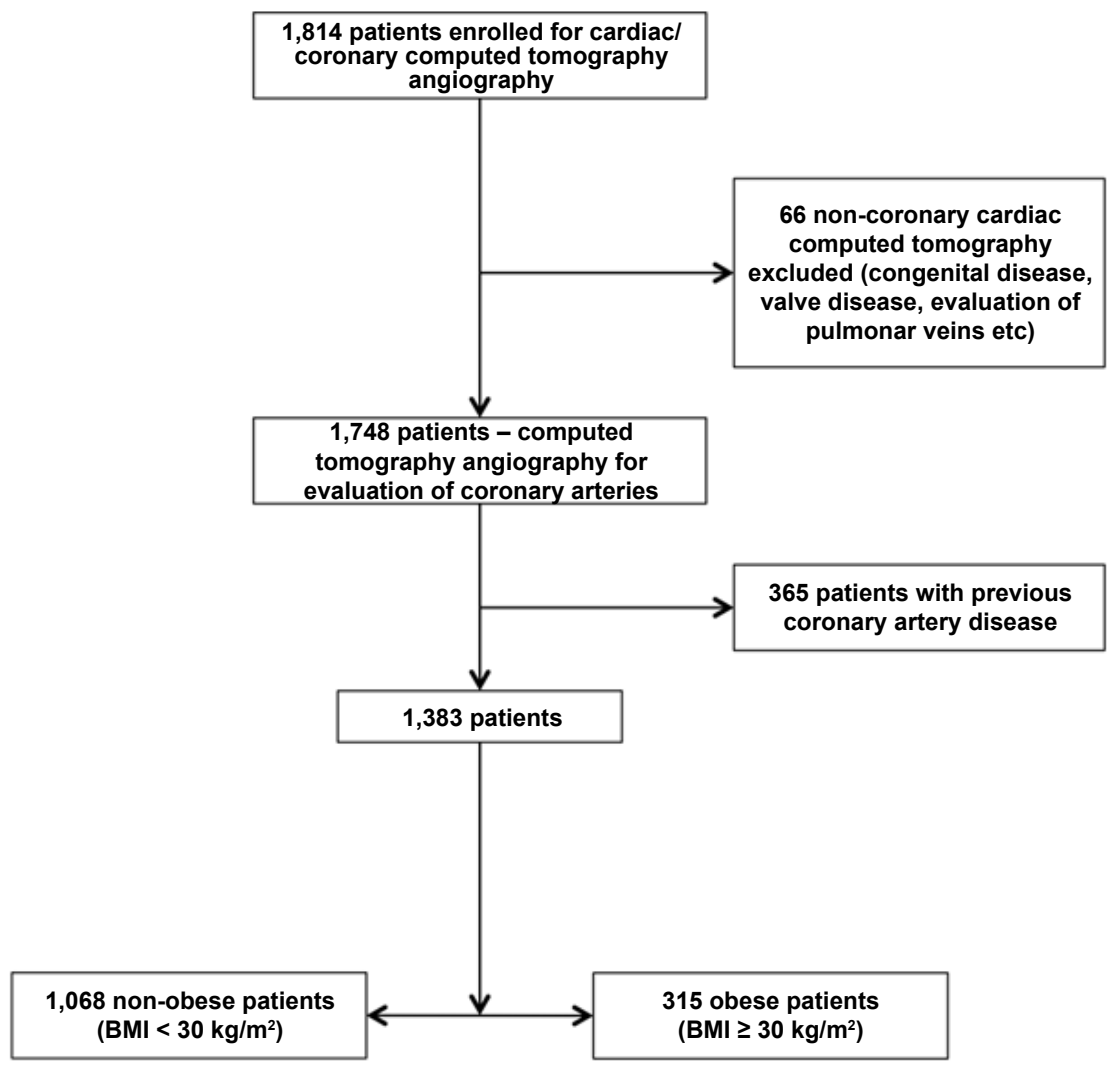

Figure 1 - Flowchart of patients' selection.

Table 1 - Characteristics of the study population

\begin{tabular}{|c|c|c|c|}
\hline Variable & Non-obese $(n=1,068)$ & Obese $(n=315)$ & $\mathbf{p}$ \\
\hline Age (years) & $58.1 \pm 11.2$ & $59.8 \pm 11.3$ & $0.02^{+}$ \\
\hline Male sex & $694(65.0 \%)$ & $223(70.8 \%)$ & $0.06^{++}$ \\
\hline Weight $(\mathrm{kg})$ & $75.8 \pm 14.0$ & $99.9 \pm 15.9$ & $<0.001^{+}$ \\
\hline Body mass index $\left(\mathrm{kg} / \mathrm{m}^{2}\right)$ & $26.2 \pm 3.4$ & $34.0 \pm 4.0$ & $<0.001^{+}$ \\
\hline Diabetes (n, \%) & $227(21.3 \%)$ & $61(19.4 \%)$ & $0.47^{++}$ \\
\hline Hypertension (n, \%) & $586(54.9 \%)$ & $184(58.4 \%)$ & $0.27^{++}$ \\
\hline Dyslipidemia (n, \%) & $413(38.7 \%)$ & $108(34.3 \%)$ & $0.16^{++}$ \\
\hline Current and previous smoking (n, \%) & $718(67.2 \%)$ & $215(68.3 \%)$ & $0.71^{++}$ \\
\hline Family history of CAD (n, \%) & $169(15.8 \%)$ & $53(16.8 \%)$ & $0.67^{++}$ \\
\hline Calcium score (median)* & 1.4 & 14.7 & $0.019^{\S}$ \\
\hline Prevalence of obstructive CAD (n, \%) & $197(18.4 \%)$ & $58(18.4 \%)$ & - \\
\hline
\end{tabular}




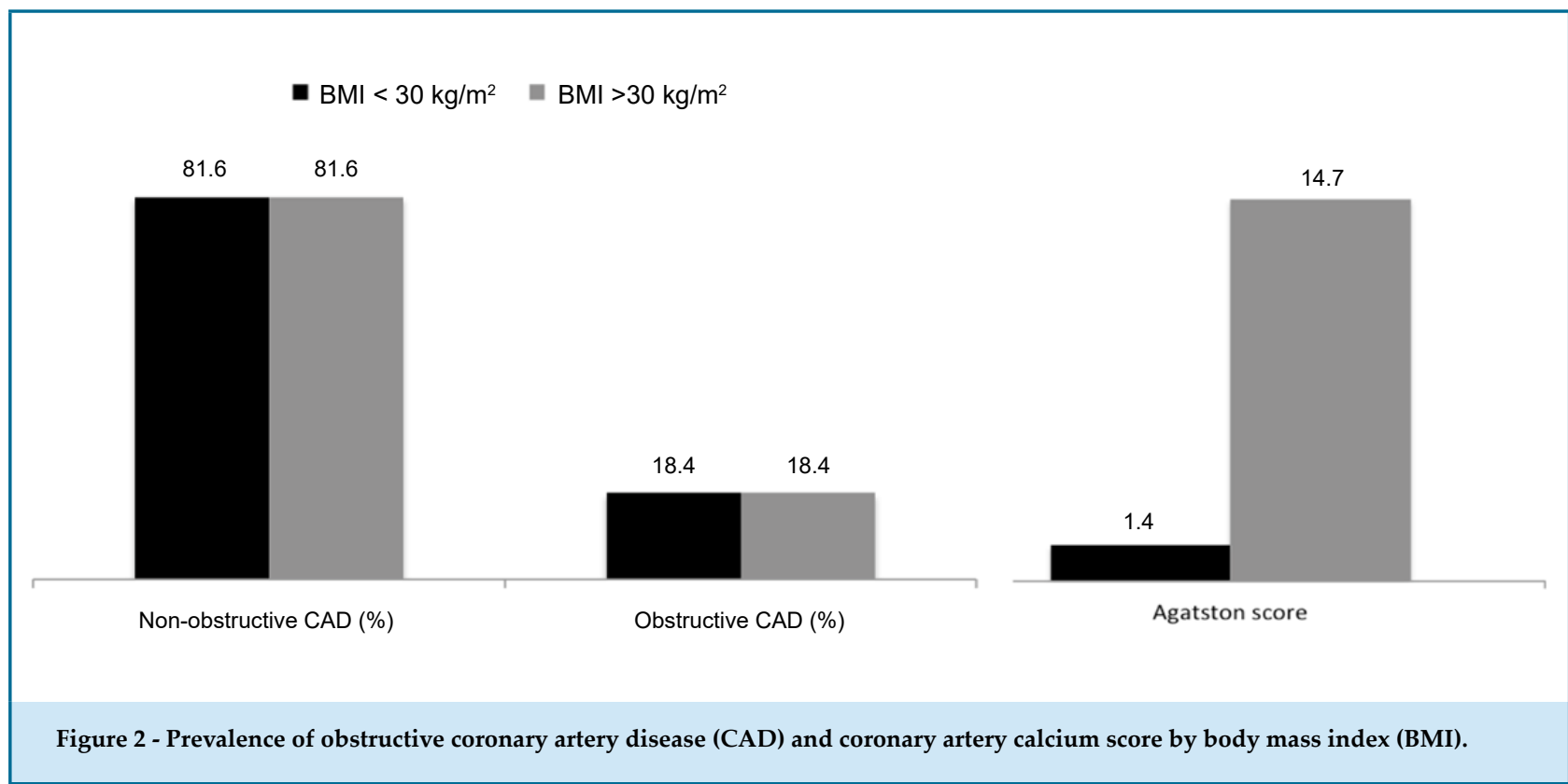

linear regression model including all cardiovascular risk factors (Table 2). Variables significantly associated with obstructive CAD, defined by CCTA, included age, male sex, and diabetes; hypertension was of marginal significance for outcome definition $(p=0.08)$. Obesity was not correlated with obstructive CAD $(p=0.10)$ when the other variables were maintained constant.

\section{Discussion}

The present study showed that, although the prevalence of obstructive CAD was not different between obese and non-obese patients, coronary artery calcium scores were significantly lower in non-obese than obese patients.

Obesity is believed to have a direct effect on metabolic health, since proinflammatory cytokines released by the adipose tissue can lead to subclinical inflammation at long-term, even if counterbalanced by anti-inflammatory cytokines. This condition is characterized by a gradual increase in inflammatory markers, such as C-reactive protein, TNF-alpha and interleukin-6, which have a direct relationship with insulin resistance, hepatic steatosis and endothelial dysfunction, leading to atherosclerosis. ${ }^{14}$

Table 2 - Obesity and risk factors as predictors of obstructive coronary arterial disease according to coronary computed tomography angiography

\begin{tabular}{lcccc}
\hline $\begin{array}{l}\text { Obstructive coronary } \\
\text { artery disease }\end{array}$ & Coef. & Standard error & 95\%CI & $\mathbf{p}^{*}$ \\
\hline Obesity & -0.035 & 0.021 & $-0.077-0.007$ & 0.102 \\
Age & 0.005 & 0.0008 & $0.003-0.0065$ & $<0.001$ \\
Sex & 0.046 & 0.01 & $0.027-0.066$ & 0.001 \\
Diabetes & 0.065 & 0.024 & $0.019-0.11$ & 0.08 \\
Hypertension & 0.034 & 0.020 & $-0.004-0.073$ & 0.548 \\
Dyslipidemia & 0.012 & 0.020 & $-0.027-0.05$ & 0.308 \\
Smoking & 0.015 & 0.015 & $-0.014-0.04$ & 0.105 \\
Family history of CAD & -0.022 & 0.013 & $-0.048-0.004$ & \\
\hline * p-values by multiple linear regression model. & & & \\
\hline
\end{tabular}


Despite the great potential of the method, the use of CCTA for the establishment of a correlation between CAD and obesity is still little explored. Compared with catheterization, computed tomography angiography is a highly accurate, non-invasive method, with acceptable levels of patient radiation and contrast, that can be useful in the identification of coronary arterial narrowing by atherosclerotic plaques.

Although the association of obesity with CAD is well documented, ${ }^{15,16}$ there is evidence supporting that cardiovascular risk factors are not more common in $\mathrm{MHO}$ individuals compared with non-obese subjects. ${ }^{17-19}$ In other words, obesity alone would not be determinant for increased incidence of CAD. This is corroborated by our findings on the prevalence of obstructive coronary disease, which was not different between obese and non-obese subjects.

On the other hand, the higher values of coronary artery calcium score among obese individuals suggest a correlation between this condition with the development of subclinical atherosclerosis. Chang et al., ${ }^{20}$ demonstrated that $\mathrm{MHO}$ patients have higher calcium score values than non-obese patients. However, after adjusting for metabolic risk factors, this association was attenuated and no longer statistically significant. The authors concluded that obesity is an additional risk for coronary atherosclerosis, including the subclinical form, mediated by metabolic changes whose thresholds are lower than those considered abnormal.

In this context, one important factor is the influence of BMI on tomography imaging analysis. Obese individuals show a reduced signal-to-noise ratio in chest images, due to increased adipose tissue compared with non-obese individuals. The higher chest wall thickness in obese subjects attenuates the X-rays emitted from the tubes, allowing that a lower amount of photons reaches the detector for image construction, resulting in a more "grained" image. Such loss could be compensated by modulations in the tube voltage and in the X-ray tube current, improving the signal-to-noise ratio of these tests. However, the methods used for image acquisition for coronary artery calcium scoring do not allow adjustments in tube voltage of the tomography scanner, fixing it at 120 kilovolts. In practical terms, that implicates that images with lower signal-to-noise ratio are obtained from CCTA in obese patients. In parallel with the potential effect of obesity on coronary calcification, we believe that this change in the signal-to- noise ratio in obese patients image may have contributed to changes in the threshold for coronary artery calcium detection, artificially increasing calcium score levels in this population. In this regard, mean calcium score percentile in our patients was 61 according to the MultiEthnic Study of Atherosclerosis (MESA), ${ }^{21}$ indicating a higher-than-average coronary calcification. However, these results are not comparable with those reported in the MESA study, which evaluated asymptomatic individuals, with not history of CAD, due to selection bias of our study population (patients referred for coronary tomography for investigation of CAD and hence more likely to have the disease).

\section{Limitations}

Our study has limitations inherent to its retrospective design. Since this was a cross-sectional study evaluating the association of obesity with CAD based on medical records, the results do not take into account some variables, such as the time of exposure to triggering factors of the disease.

The definition of metabolically healthy obesity was based on the identification and exclusion of obesity-related metabolic abnormalities (hypertension, dyslipidemia, diabetes). Nevertheless, laboratory markers of insulin resistance, including the Homeostatic Model Assessment of Insulin Resistance (HOMA-IR) were not used in patients recruitment in our study.

Our study population was selected based on BMI, which, although is the most widely used anthropometric variable to characterize obesity, it does not provide information regarding body composition. Therefore, assuming that the percentage of body fat has a direct effect on insulin resistance, BMI alone does not give us any insight into this condition. In addition, other anthropometric measures known to provide a more accurate estimation of visceral fat (e.g. waist circumference and waist-to-hip ratio measurements) were not registered in the medical records, and hence could not be used in the analysis.

Finally, the definition of CAD by CCTA may be controversial; although CCTA is a very robust method to define non-obstructive atherosclerosis by coronary artery calcium scoring, the method considered the goldstandard method to detect obstructive coronary disease is invasive coronary angiography combined or not with intracoronary ultrasound. 


\section{Conclusion}

Obese patients without other associated risk factors did not show a higher prevalence of obstructive CAD, according to CCTA, compared with non-obese patients. However, coronary artery calcium score was higher in obese than in non-obese individuals, indicating a higher prevalence of subclinical atherosclerosis mediated by obesity.

\section{Author contributions}

Conception and design of the research: Pereira LLS, Moraes GM, Carneiro ACC, Moreira VM, Bello JHSM, Prazeres CEE, Rochitte CE, Magalhaes T. Acquisition of data: Pereira LLS, Moraes GM, Carneiro ACC, Moreira VM, Bello JHSM, Prazeres CEE, Rochitte CE, Magalhaes T. Analysis and interpretation of the data: Pereira LLS, Moraes GM, Rochitte CE, Magalhaes T. Statistical analysis: Pereira LLS, Moraes GM, Magalhaes T. Writing of the manuscript: Pereira LLS, Moraes GM, Magalhaes T. Critical revision of the manuscript for intellectual content: Pereira LLS, Moraes GM, Carneiro ACC, Moreira VM, Bello JHSM, Prazeres CEE, Rochitte CE, Magalhaes T.

\section{References}

1. GBD 2016 Causes of Death Collaborators. Global, regional, and national age-sex specific mortality for 264 causes of death, 1980-2016: a systematic analysis for the Global Burden of Disease Study 2016. Lancet. 2017;390(10100):1151-210.

2. World Health Organization.(WHO). Global status of noncommunicable diseases. Geneva; 2014.

3. Arnlov J, Ingelsson E, Sundstrom J, Lind L. Impact of body mass index and the metabolic syndrome on the risk of cardiovascular disease and death in middle-aged men. Circulation.2010;121(2):230-6.

4. Karelis AD. To be obese-does it matter if you are metabolically healthy? Nat Rev Endocrinol. 2011;7(12):699-700.

5. BlüherM. Are there still healthy obese patients? Curr Opin Endocrinol Diabetes Obes. 2012;19(5):341-6.

6. Després JP. What is "metabolically healthy obesity?": from epidemiology to pathophysiological insights. J Clin Endocrinol Metab. 2012;97(7):2283-5.

7. Primeau V, L. Coderre, A.D. Karelis, Brochu M, Lavoie ME, Messler V, et al. Characterizing the profile of obese patients who are metabolically healthy, Int J Obes (Lond).2011;35(7):971-81.

8. Wildman RP, Muntner P, Reynolds K, McGinn AP, Rajpathak S, WylieRosett J, et al. The obese without cardiometabolic risk factor clustering and the normal weight with cardiometabolic risk factor clustering: prevalence and correlates of 2 phenotypes among the US population (NHANES 1999-2004), Arch Intern Med. 2008;168(15):1617-24.

\section{Potential Conflict of Interest}

The authors have declared that no competing interests exist.

\section{Sources of Funding}

There were no external funding sources for this study.

\section{Study Association}

This article is part of academic works submitted by Lara Luiza Silvello Pereira and Gisele Marochi de Moraes in partial fulfillment of the requirements for the Bachelor's degree in Medicine, from Pontifícia Universidade Católica do Paraná.

\section{Ethics approval and consent to participate}

This study was approved by the Ethics Committee of the Pontifícia Universidade Católica do Paraná under the protocol number 1.524.216. All the procedures in this study were in accordance with the 1975 Helsinki Declaration, updated in 2013. Informed consent was obtained from all participants included in the study.

9. Kramer CK, Zinman B, Retnakaran R. Are metabolically healthy overweight and obesity benign conditions?: A systematic review and meta-analysis, Ann Intern Med.2013;159(11):758-69.

10. Meigs JB, Wilson PW, Fox CS, Vassan RS, Nathan DM, Sullivan LM, et al. Body mass index, metabolic syndrome, and risk of type 2 diabetes or cardiovascular disease. J Clin Endocrinol Metab. 2006;91(8):2906-12.

11. Yeboah J, McClelland RL, Polonsky TS, Burke GL, Sibley CT, O'Leary D. Comparison of novel risk markers for improvement in cardiovascular risk assessment in intermediate-risk individuals. JAMA. 2012;308(8):788-95.

12. Agatston AS, Janowitz WR, Hildner FJ, Zusmer NR, Viamonte M Jr, Detrano R. Quantification of coronary artery calcium using ultrafast computed tomography. J Am Coll Cardiol. 1990;15(4):827-32.

13. Raff GL, Abidov A, Achenbach S, Berman DS, Boxt LM, Budoff MJ, et al. SCCT guidelines for the interpretation and reporting of coronary computed tomographic angiography. J Cardiovasc Comput Tomogr. 2009;3(2):122-36

14. Alkhawam H, Nguyen J, Sayanlar J, Sogomonian R, Desai R, Jolly J, et al Coronary artery disease in patients with body mass index ] $30 \mathrm{~kg} / \mathrm{m} 2$ : a retrospective chart analysis. J Community Hosp Intern Med Perspect. 2016;6(3):31483.

15. McGill HC, McMahan CA, Herderick EE, Zieske AW, Malcom GT Tracy RE, et al. Pathobiological determinants of atherosclerosis in youth (PDAY) Research Group. Obesity accelerates the progression of coronary aterosclerosis in young men. Circulation. 2002;105(23):2712-8. 
16. Guh DP, Zhang W, Bansback N, Amarsi Z, Birmingham CL, Anis AH. The incidence of co-morbidities related to obesity and overweight: a systematic review and meta-analysis. BMC Public Health. 2009 Mar $25 ; 9: 88$

17. St-Pierre AC, Cantin B, Mauriège P, Bergeron J, Dagenais GR, Després $\mathrm{JP}$, et al.et al. Insulin resistance syndrome, body mass index and the risk of ischemic heart disease. CMAJ. 2005;172(10):1301-5.

18. Meigs JB, Wilson PW, Fox CS, Vasan RS, Nathan DM, Sullivan LM, et al. Body mass index, metabolic syndrome, and risk of type 2 diabetes or cardiovascular disease. J Clin Endocrinol Metab. 2006;91(8):2906-12.
19. Hamer M, Stamatakis E. Metabolically healthy obesity and risk of allcause and cardiovascular disease mortality. J Clin Endocrinol Metab. 2012;97(7):2482-8

20. Chang Y, Kim BK, Yun KE, Cho J, Zhang Y, Rampal S, et al. Metabolicallyhealthy obesity and coronary artery calcification. J Am Coll Cardiol. 2014; 63(24):2679-86.

21. Detrano R, Guerci AD, Carr JJ, Bild DE, Burke G, Folsom AR, et al. Coronary calcium as a predictor of coronary events in four racial or ethnic groups. N Engl J Med. 2008; 358(13):1336-45. 